\title{
Optimal Control of Quasilinear Elliptic Equations with non Differentiable Coefficients at the Origin
}

\author{
EDUARDO CASAS and LUIS AIBERTO FI:RNÁNDI:Z
}

\begin{abstract}
In this paper we study some optimal control problems of systems governed by quasilinear elliptic equations in divergence form with non differentiable coefficients at the origin. We prove existenee of solutions and derive the optimality conditions by considering a perturbation of the differential operator coefficients that removes the singularity at the origin. Regularity of optimal controls is also deduced
\end{abstract}

\section{INTRODUCTION}

We will be considering optimal control problems involving the differential operator

$$
A_{y}:=-\operatorname{div}\left(\varphi(x,|\nabla, y|) \nabla_{y}\right)+\psi(x, y)
$$

with $\varphi: \Omega \times(0,+\infty) \rightarrow(0,+\infty)$ and $\psi: \Omega \times \mathfrak{R} \rightarrow \mathcal{R}$, where $\Omega$ is a bounded open subset of $\mathcal{R}^{N}$ with Lipschitz continuous boundary $\mathrm{I}$.

Authors have studied control problems associated with quasilinear elliptic operators in $[2,3,5]$. The novelty of this work is that the non differentiability of $\varphi(x,$.$) at 0$ is allowed, which causes the non differentiability of state with respect to the control. This is not an obstacle to prove existence of optimal controls. but it becomes complicated to derive the optimality conditions. To overcome this difficulty, we introduce a family of approximating control problems that fall in the class of problems treated in [3.5]. 
Opcrator $A$ introduced above is less general than those ones studied in $[2,3,5]$. Nevertheless, most of the known quasilinear elliptic operators belong to this class. Next, we establish the hypotheses on $A$.

Let $b: \Omega \times[0,+\infty) \rightarrow[0,+\infty)$ be the function defined by

$$
b(x, s)=\varphi(x, s) s
$$

We will assume the following conditions

$$
\begin{aligned}
& b \in C(\Omega \times[0,+\infty)) \cap C^{\prime}(\Omega \times(0,+\infty)) \\
& \left\{\begin{array}{l}
\psi(\ldots s) \text { is a measurable function on } \Omega \\
\psi(x, .) \text { belongs to } C^{\prime}(\Re)
\end{array}\right. \\
& \Lambda_{1}(k+s)^{\alpha-2} \leq \frac{\partial b}{\partial s}(x, s) \leq \Lambda_{2}(k+s)^{n-2} \quad \forall s \in(0,+\infty) \\
& \sum_{i=1}^{n}\left|\frac{\partial b}{\partial x_{i}}(x, s)\right| \leq \Lambda_{2}(k+s)^{r-2} s \quad \forall s \in(0,+\infty) \\
& 0 \leq \frac{\partial \psi}{\partial s}(x, s) \leq f(|s|) \quad \forall s \in \mathfrak{R} \\
& b(x, 0)=\psi(x, 0)=0
\end{aligned}
$$

for some $k \in[0,1]$ : some $\alpha \in(1,+\infty)$, some strictly positive constants $A_{1}, A_{2}$, some positive and non decreasing function $f$ and a. e. $x \in \Omega$.

Let us consider the boundary value problem:

$$
\begin{cases}A_{V}=\nu & \text { in } \Omega \\ v=0 & \text { on } I\end{cases}
$$

We make the following additional assumption on $\alpha$

$$
\alpha>\dot{N} / 2
$$

In the sequel. $W-1 . /(\Omega)$ will denote the dual of the usual Sobolev space $W^{\prime}$ " (IL) $\left(\frac{1}{\alpha}+\frac{1}{\beta}=1\right)$ and (...) their duality product. Also, $D(\Omega)$ will denote the space of infinitely differentiable functions with a compact support in $\Omega$. 
We will prove that problem (1.8) has a unique solution $y_{1} \in$ $W_{0}^{\prime-\alpha}(\Omega) \cap L^{\infty}(\Omega)$ for each $\nu \in L^{2}(\Omega)$. Hypothesis (1.9) is essential to deduce the boundedness of the solution $v_{v}$.

\section{Remark:}

Hypothesis (1.7) can be weakened in the following form:

$$
\psi(, .0) \in L^{2}(\Omega)
$$

In this case it is enough to do the change

$$
\bar{\psi}(x, s)=\psi(x, s)-\psi(x, 0)
$$

Let us give some examples of the principal part of operators $A$ that satisfy' previous hypotheses with $b \notin C^{\prime}(\Omega \times[0,+\infty))$ :

Example 1.-(Case $\alpha \geq 2, k \neq 0)$

$$
\varphi(x, s)=\lambda(x)+\sin (\ln s)+s^{(x-2}
$$

with $\lambda \in C^{\prime}(\bar{\Omega})$ such that $2<\lambda(x) \quad \forall x \in \bar{\Omega}$.

Example 2.-(Case $\alpha<2, k=0$ )

$$
\varphi(s)=s^{n-2}
$$

Example 3.-(Case $\alpha<2, k \neq 0$ )

$$
\varphi(x, s)=\sin ^{3}|\ln s|^{12} \cdot \exp (-s)+\lambda(x)(k+s)^{\alpha-2}
$$

with $\lambda \in C^{\prime}(\bar{\Omega})$ such that $0<\lambda_{0}<\lambda(x) \quad \forall x \in \Omega$ for a sufficiently large constant $\lambda_{0}$.

If $\alpha>2$ and $k=0$, it follows easily from $(1.2),(1,5),(1,4)$ and $(1.7)$ that $b \in C^{\prime}(\Omega \times[0,+\infty))$ with $\frac{\partial b}{\partial s}(x, 0)=0$. Therefore, the operator $A$ satisfies the hypotheses of papers [3.5], as we will see later (lemma 2.2). 
Let us introduce the following optimal control problem:

$$
\left(P_{r r}\right)\left\{\begin{array}{l}
\text { Minimize } J(\nu) \\
\nu \in \mathbb{K}
\end{array}\right.
$$

where $\mathcal{H}$ is a non empty, convex and closed subset of $L^{2}(\Omega)$ and $J: L^{2}(\Omega) \rightarrow \mathcal{R}$ is the functional defined by

$$
f(v)=\frac{1}{2} \int_{n}\left|v_{n}-y\right|^{2} d x+\frac{\rho}{2} \int_{n}|v|^{2} d x
$$

with $y_{d}$ a fixed element of $L^{2}(\Omega)$ and $\rho$ a non negative constant. Let us remark that thanks to (1.9). it is verified the continuous imbedding

$$
L^{2}(\Omega) \subset W^{-1} \cdot \beta(\Omega)
$$

\section{Remark}

If we suppress $(1.9)$, it is possible to carry out the study of $\left(P_{(z)}\right)$, substituting ( 1.6$)$ by condition

$$
0 \leq \frac{\partial \psi}{\partial s}(x, s) \leq \Lambda_{2}(k+|s|)^{x-2}
$$

for all $x \in \Omega$ and all $s \in \mathcal{R}$.

Nevertheless. for $1<\alpha \leq \frac{2 N}{N+2}$ and $N \geq 3$, we must formulate $\left(P_{w}\right)$ in a slight different form. Variations are motivated by the lact that in this case $W_{1}^{\prime}$ " $(\Omega)$ is not imbedded in $l^{2}(\Omega)$ (see Casas and Fernández [2]).

The plan of the paper is as follows: in next section, we state some auxiliary lemmas about the differential operator and state equation; in Section 3, we prove existence of solutions and formulate the optimality necessary conditions for $\left(P_{\alpha}\right)$; Sections 4 and 5 are devoted to the proof of these optimality conditions; in last section, we obtain $H^{\prime}(\Omega)$ regularity (resp. $W^{\prime \prime}$ a $(\Omega)$ if $\alpha<2$ ) for optimal controls.

\section{SOME AUXILIARY LEMMAS}

In this section. we prove existence, uniqueness and contimuous dependence of solutions of Dirichlet problem associated with operator $A$ as well as some 
perturbed operators $A_{\varepsilon}$. We begin showing some properties about the coefficients:

Lemma 2.1. Let us suppose (1.2), (I.4) and $b(x, 0)=0$. Then, there are posirive constants $\Lambda_{3}$ and $\Lambda_{4}$ depending only on $\alpha, \Lambda_{l}$ and $\Lambda_{2}$ such that

$$
\Lambda_{3}(k+s)^{\alpha-2} \leq \varphi(x, s) \leq \Lambda_{4}(k+s)^{\alpha-2} \quad \forall x \in \Omega, \quad \forall s \in(0,+\infty)
$$

\section{Proof}

Using $(1,2)$ and the fact that $b(x, 0)=0$, we get

$$
\varphi(x, s) s=b(x, s)=\int_{0}^{s} \frac{\partial b}{\partial s}(x, l) d t
$$

In virtue of (1.4), we have that

$$
\Lambda_{1} \int_{0}^{s}(k+t)^{\alpha-2} a t \leq \int_{0}^{n} \frac{\partial b}{\partial s}(x, t) d t \leq \Lambda_{2} \int_{0}^{a}(k+t)^{\alpha-2} d t
$$

If $\alpha \geq 2$, it is clear that

$$
\begin{gathered}
\Lambda_{!} \int_{0}^{1}(k+t)^{\alpha-2} d t=\Lambda_{1} \frac{(k+s)^{\alpha-1}-k^{\alpha-1}}{\alpha-1}= \\
=\Lambda_{1} \frac{k(k+s)^{\alpha-2}-k^{\alpha-1}+s(k+s)^{\alpha-2}}{\alpha-1} \geq \frac{\Lambda_{1}}{\alpha-1}(k+s)^{\alpha-2} s .
\end{gathered}
$$

If $\alpha \leq 2$, applying the mean value theorem, it follows that

$$
\Lambda_{1} \frac{(k+s)^{\alpha-1}-k^{\alpha-1}}{\alpha-1}=\Lambda_{1}(k+\theta s)^{\alpha-2} s \geq \Lambda_{1}(k+s)^{\alpha-2} s
$$

because $\theta \in(0,1)$.

In any case, we obtain that

$$
\Lambda_{3}(k+s)^{r-2} \leq \varphi(x, s) \quad \forall x \in \Omega, \quad \forall s \in(0,+\infty)
$$


with $\Lambda_{3}=\frac{\Lambda_{1}}{\alpha-1}($ if $\alpha \geq 2)$ and $\Lambda_{3}=\Lambda_{1}$ (if $\left.\alpha \leq 2\right)$.

For the upper bound. let us remark that if $\alpha \geq 2$, it is verified that

$$
\Lambda_{2} \int_{11}^{\prime}(k+t)^{r-2} d t \leq \underline{\Lambda}_{2}(k+s)^{r-2} s
$$

and if $\alpha \leq 2$,

$$
A_{2} \int_{11}^{\alpha}(k+t)^{\alpha-2} d t=\Lambda_{2} \frac{(k+s)^{\alpha-1}-k^{(a-1}}{\alpha-1} \leq \frac{\lambda_{2}}{\alpha-1}(k+s)^{\alpha-2} s
$$

Thus, we deduce that $\Lambda_{4}=\frac{\Lambda_{2}}{\alpha-1}$ (if $\left.\alpha \leq 2\right)$ and $\Lambda_{4}=\Lambda_{2}$ (if $\alpha \geq 2$ ).

Next. we introduce the perturbed differential operator coefficients and state the coercivity and growth conditions.

Lemma 2.2. Let us suppose (1.2), (1.4), (1.5) and $b(x, 0)=0$. For each $\varepsilon \geq 0$, let us introduce $a^{2}: \Omega \times \mathfrak{R}^{N} \rightarrow \mathfrak{R}^{N}$ defined by

$$
a^{2}(x, \eta)=\varphi(x, c+|\eta|) \eta
$$

Then, $a^{2} \in C^{\prime}\left(\Omega \times \mathcal{R}^{N}\right)$ if $\varepsilon>0$ and $a^{0} \in C\left(\Omega \times \mathcal{R}^{N}\right) \cap C^{1}\left(\Omega \times\left(\mathbb{R}^{N} \backslash\{0\}\right)\right)$.

Moreover, there exist a pessitive constant $\Lambda_{5}$ depending only an $N, \alpha, \Lambda$, and 1. serth shost

$$
\begin{aligned}
& \sum_{i, i}^{\sum_{i}} \frac{\partial a_{i}^{e}}{\partial \eta_{i}}(x, \eta) \xi_{i} \xi_{i} \geq \lambda_{3}(k+\varepsilon+|\eta|)^{2}=2|\xi|^{2} \\
& \sum_{i, j}^{n}\left|\frac{\partial a_{i}^{l}}{\partial \eta_{i}}(x, \eta)\right| \leq \lambda_{5}(k+\varepsilon+|\eta|)^{x^{2}}= \\
& \sum_{i, j}^{N}\left|\frac{\partial a_{i}^{r}}{\partial x_{i}}(x, \eta)\right| \leq N d_{2}(k+\varepsilon+|\eta|)^{n}{ }^{2}|\eta|
\end{aligned}
$$

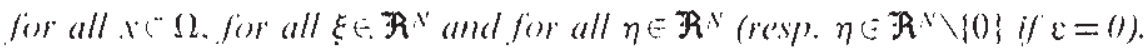




\section{Proof.}

Given $i, j \in\{1, \ldots, N\}$, it is immediate to verify that

$$
\frac{\partial a_{i}^{\varepsilon}}{\partial \eta_{i}}(x, \eta)=\varphi(x, \varepsilon+|\eta|) \delta_{i j}+\frac{\partial \varphi}{\partial s}(x, \varepsilon+|\eta|) \frac{\eta_{i} \eta_{i}}{|\eta|}
$$

Therefore, we have that

$$
\begin{aligned}
& \frac{\partial a_{j}^{e}}{\partial \eta_{i}}(x, \eta) \in C\left(\Omega \times \mathcal{H}^{N^{\prime}}\right) \text { if } \varepsilon>0\left(\text { resp. } C\left(\Omega \times\left(\mathcal{R}^{N} \backslash\{0\}\right)\right) \text { if } \varepsilon=0\right) \text { and } \\
& \sum_{i, j=1}^{N} \frac{\partial a_{i}^{\varepsilon}}{\partial \eta_{i}}(x, \eta) \xi_{i} \xi_{i}=\varphi(x, \varepsilon+|\eta|) \mid \xi^{2}+\frac{\partial \varphi}{\partial s}(x, \varepsilon+|\eta|) \frac{\left(\eta^{T} \xi\right)^{2}}{|\eta|}
\end{aligned}
$$

Let us suppose that $\frac{\partial \varphi}{\partial s}(x . c+|\eta|) \geq 0$. Hence.

$$
\sum_{i, j=1}^{k} \frac{\partial a_{i}^{\varepsilon}}{\partial \eta_{i}}(x, \eta) \xi_{i} \xi_{j} \geq \varphi(x, \varepsilon+|\eta|)|\xi|^{2} \geq \Lambda_{3}(k+\varepsilon+|\eta|)^{a-2}|\xi|^{2}
$$

thanks to lemma 2.1 .

Otherwise, if $\frac{\partial \varphi}{\partial x}(x, \varepsilon+|\eta|)<0$. using the Catuchy - Schwarz inequality and (1.4), we get

$$
\sum_{i . j}^{\prime \prime} \frac{\partial a_{i}^{\mathrm{r}}}{\partial \eta_{i}}(x, \eta) \xi_{i} \xi_{i} \geq\left(\varphi(x, \varepsilon+|\eta|)+\frac{\partial \varphi}{\partial s}(x, \varepsilon+|\eta|)|\eta|\right)|\xi|^{2} \geq
$$

$\geq \frac{\partial b}{\partial s}(x, \varepsilon+|\eta|)|\xi|^{2} \geq \Lambda_{1}(k+\varepsilon+|\eta|)^{*}{ }^{2}|\xi|^{2} \geq \Lambda_{3}(k+\varepsilon+|\eta|)^{\prime}=|\xi|^{2}$.

Belore proving second inequality of lemma, let us note that from (1.4) and lemma 2.1, it follow's

$$
\left|\frac{\partial \varphi}{\partial s}(x, s) s\right|=\left|\frac{\partial b}{\partial s}(x, s)-\varphi(x, s)\right| \leq\left(\Lambda_{2}+\Lambda_{+}\right)(k+. s)^{*} \quad 2 \forall x \in \Omega, \forall s(\cdot(0,+\infty)
$$

Now. combining this expression with formula $(2.1)$, we deduce

$$
\sum_{i, i}^{N}\left|\frac{\partial a_{i}^{r}}{\partial \eta_{i}}(x, \eta)\right| \leq N\left(|\varphi(x, \varepsilon+|\eta|)|+|\eta|\left|\frac{\partial \varphi}{\partial s}(x, \varepsilon+|\eta|)\right|\right) \leq
$$




$$
\leq \lambda_{5}(k+\varepsilon+|\eta|)^{n-2} \quad \text { where } \lambda_{5}=N\left(2 \lambda_{4}+\lambda_{2}\right)
$$

Latst inequality of lemma follows directy from (1.5).

Lemma 2.3. Let us suppose (1.2)-(1.4), (1.6) and (1.7). Then, there exist positive constants $\Lambda_{6}$ and $\Lambda_{7}$ depending only on $N, \alpha, \Lambda_{1}$ and $\Lambda_{2}$ such that

a) $\sum_{i}^{n}\left(a_{i}^{c}(x, \eta)-a_{j}^{c}\left(x, \eta^{\prime}\right)\right)\left(\eta_{i}-\eta_{j}^{\prime}\right) \geq L_{6} \begin{cases}\left(1+|\eta|+\left|\eta^{\prime}\right|\right)^{n-2}\left|\eta-\eta^{\prime}\right|^{2} & \text { if } \alpha \leq 2 \\ \left|\eta-\eta^{\prime}\right|^{\prime \prime} & \text { if } \alpha \geq 2\end{cases}$

b) $\quad \sum_{i=1}^{n}\left|a_{i}^{L}(x, \eta)\right| \leq \Lambda_{7}(k+|\eta|)^{n+2}|\eta|$

Furhermore.

c) $\quad\left(\psi(x, s)-\psi\left(x, x^{\prime}\right)\right)\left(s-x^{\prime}\right) \geq 0$

(1) $\quad|\psi(x, s)| \leq|s| / f(|s|)$

for $\varepsilon \geq 0$, for all $x \in \Omega$, all $s, r^{\prime} \in \mathcal{R}$ and all $\eta, \eta^{\prime} \in \mathcal{R}^{*}$.

\section{Proof}

For a) and b). see lemma 1 of Tolksdorf [13]. Conditions c) and d) follow immediately from the hypotheses.

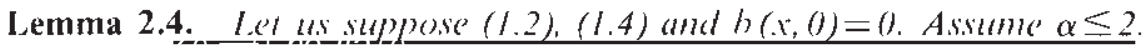
$7 h^{2} n$, for eache $\geq 0$ we have

(a) $\int_{12}\left(a^{2}\left(x, \nabla y^{\prime}\right)-a^{r}\left(x, \nabla y^{\prime \prime}\right)\right)\left(\nabla !-\nabla y^{\prime}\right) d x \geq$

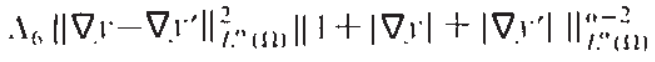

Moreover, there exist positive constants $\lambda_{N}$ and $\lambda_{y}$ depending onl! on $N$. $\alpha, 1_{1}$ and $1_{2}$.stekth that

b)

$$
\sum_{i=1}^{x} a_{j}^{2}(x, \eta) \eta_{j} \geq\left\{\begin{array}{l}
\lambda_{x}|\eta|^{n}-\lambda_{9} \\
A_{x}\left(|\eta|^{\prime \prime}-|\eta|\right)
\end{array}\right.
$$

for $v \geq 0$. for all $x \in \Omega$ and all $\eta \in \mathcal{R} x$. 


\section{Proof}

a) It is a simple consequence of Lemma 2.3-a) and Hölder's inequality applied with $p=2 / \alpha$ and $p^{\prime}=2 /(2-\alpha)$ :

$$
\begin{gathered}
\int_{\Omega 2}\left|\nabla y^{\prime}-\nabla y^{\prime}\right|^{\alpha} d x \leq \\
\leq\left(\int_{\Omega 2}\left|\nabla y-\nabla y^{\prime}\right|^{2}\left(1+|\nabla y|+\left|\nabla y^{\prime}\right|\right)^{(x-2} d x\right)^{k 2}\left(\int_{\Omega}\left(1+|\nabla y|+\left|\nabla y^{\prime}\right|\right)^{\alpha} d x\right)^{(2-(x) 2}
\end{gathered}
$$

b) It is enough to take into account Lemma 2.3-a) again, hypothesis $(1.7)$ and to distinguish the cases $|\eta| \geq 1$ and $|\eta|<1$.

Now, we are ready to derive existence and uniqueness of solution for the Dirichlet problems. First, for each $\varepsilon>0$, let us introduce the perturbed differential operator

$$
A_{\mathrm{c},} y^{\prime}=-\operatorname{div}\left(a^{2}(x, \nabla y)\right)+\psi(x, y)=-\operatorname{div}(\varphi(x, \varepsilon+|\nabla y|) \nabla y)+\psi(x, y)
$$

and the correponding Dirichlet problems

$$
\left\{\begin{array}{c}
A_{\mathrm{L} .} y=\nu \text { in } \Omega \\
y=0 \text { on } \mathrm{T}
\end{array}\right.
$$

Utilizing previous lemmas $2.2-2.4$, we can apply the result of Rakotoson [10] to deduce that, given $\nu \in L^{2}(\Omega)$, there exists a unique $l_{\varepsilon}(\nu) \in$ $W_{1}^{1}{ }^{\alpha}(\Omega) \cap L^{\infty}(\Omega)$ solution of $(2,2)$ for each $\varepsilon>0$ (resp. there exists a unique $y_{1} \in W_{0}^{\prime * x}(\Omega) \cap L^{\infty}(\Omega)$ solution of $(1.8)$, for $\varepsilon=0$ )

In the following result, we show continuous dependence with respect to the data for this type of equations.

Lemma 2.5. Let us suppose (1.2)-(1.4), (1.6)-(1.7) and (1.9). Given $\varepsilon \geq 0$. let $\mathrm{l}_{\mathrm{c}} \in W_{0}^{1, e z}(\Omega)$ be the solution of

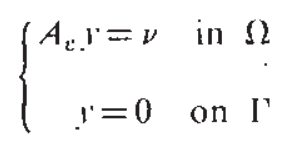

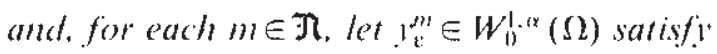

$$
\left\{\begin{array}{cc}
A_{\mathrm{L}} \cdot \mathrm{l}=v_{\mu r} & \text { in } \Omega \\
\mathrm{r}=0 & \text { on } \mathrm{J}
\end{array}\right.
$$


with $A_{i}=$ A. Assume that $v_{m}-v$ weakt! in $L^{\prime}(\Omega)$ as $m \rightarrow+\infty$.

Then, $y_{c}^{\prime n} \rightarrow y_{1}^{\prime}$ in $W_{0}^{1, n}(\Omega)$ as $m \rightarrow+\infty$.

\section{Proof}

First, let us remark that $\nu_{m} \rightarrow \nu$ in $W^{-1, \beta}(\Omega)$, because $L^{?}(\Omega) \subset W^{-1, \beta}(\Omega)$ with compact imbedding (Adams [1]).

From the relations satislied by $y_{\varepsilon}$ and $y_{c}^{\prime \prime \prime}$, it follows that

$$
\begin{aligned}
& \int_{1 !}\left(\psi\left(x, y_{c}^{\prime \prime \prime}\right)-\psi\left(x, y_{c}\right)\right)\left(y_{\varepsilon}^{\prime \prime \prime}-y_{\varepsilon}\right) d x+\int_{\Omega !}\left(a^{\varepsilon}\left(x, \nabla l_{\varepsilon}^{\prime \prime \prime}\right)-\right. \\
& a^{k}\left(x . \nabla y_{2}\right)\left(\nabla_{1}^{\prime \prime \prime}-\nabla y_{2}\right) d x=\left(\nu_{m}-\nu_{1} y_{\varepsilon}^{m \prime}-y_{2}\right)
\end{aligned}
$$

Suppose $\alpha \geq 2$. Applying a) and c) of lemma 2.3, we get

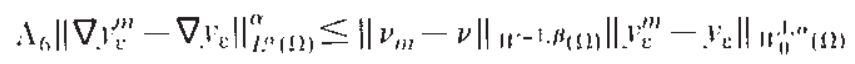

Finally, using the equivalent norm in $W_{11}^{1.9}(\Omega)$ (see [1]) and the hypotheses. we obtain

$$
l_{2}^{\prime \prime \prime} \rightarrow y_{i} \text { in } W_{n}^{\prime \prime c o}(\Omega)
$$

In the vase $\alpha<2$, argumentation is similar using lemma 2.4-il).

\section{EXISTENCE OF SOLUTION AND OPTIMALITY \\ CONDITIONS}

We begin showing existence of solutions of problem $\left(P_{c k}\right)$ defined in $(1.10)-(1.11):$

Theorem 3.1. Let us suppose (1.2)-(1.4), (1.6)-(1.7) and (1.9). Assume thas

Fither $\mathfrak{F}$ is bouthded in $l^{2}(\Omega)$ or $\rho>0$.

The'n, wore erists (at least) one solution of $\left(P_{a}\right)$. 


\section{Proof}

Let $\left\{u_{n}\right\}_{n \in \pi} \subset \mathcal{K}$ be a minimizing sequence and $\left\{z_{n}\right\}_{n \in \pi}$ the sequence of associated states. By the hypothesis, there exists $\bar{u} \in \mathcal{K}$ and a subsequence (again denoted by $\left\{u_{n}\right\}$ ) such that

$$
u_{n} \rightarrow \bar{u} \text { weakly in } L^{2}(\Omega)
$$

$\mathcal{K}$ is a convex and closed subset of $L^{2}(\Omega)$. Therefore, $\mathscr{K}$ is weakly closed in $L^{2}(\Omega)$ and $\bar{u} \in \mathcal{K}$.

Let $\bar{v}$ be the associated state of $\bar{u}$. From lemma 2.5 , we obtain that

$$
y_{m} \rightarrow \bar{l} \text { in } W_{0}^{\mid \alpha r}(\Omega)
$$

The lower semicontinuity of $J$ in the weak topology of $L^{2}(\Omega)$ and the imbedding $W_{0}^{1 . *}(\Omega) \subset L^{2}(\Omega)$, completes the proof.

Optimality conditions for problem $\left(P_{n}\right)$ can be formulated as follows:

Theorem 3.2. Let us suppose (1.2)-(1.7) and (1.9) with $k \neq 0$ if $\alpha>2$. Assume that $\mathcal{K}$ is a bounded subset of $L^{\infty}(\Omega)$. Let $\bar{u}$ be a wolution of $\left(P_{n}\right), \bar{r}$ the associated state and $\Omega_{n}=\{x \in \Omega:|\nabla \bar{v}(x)|>0\}$. Then, there exists $\bar{p} \in H_{i j}^{\prime}(\Omega)\left(\right.$ resp. $W_{0,}^{l}, \alpha(\Omega)$ if $\left.\alpha<2\right)$ such shat

$$
\begin{aligned}
& \left\{\begin{aligned}
-\operatorname{div}(\varphi(x,|\nabla \bar{y}| \nabla \bar{y}))+\psi(x, \bar{y})=\bar{u} & \text { in } \Omega \\
\bar{y}=0 & \text { on }[
\end{aligned}\right. \\
& -\operatorname{div}\left(\left(\varphi(x,|\nabla \bar{y}|) l+\frac{\partial \varphi}{\partial x}(x,|\nabla \bar{y}|) \frac{\nabla \bar{y} \cdot \nabla \bar{y} r}{|\nabla \bar{y}|}\right) \nabla \bar{p}\right)+ \\
& +\frac{\partial \psi}{\partial s}(x, \bar{y}) \bar{p}=\bar{y}-y_{1} \quad \text { in } \Omega_{0} \\
& \int_{\Omega}(\bar{p}+\rho \bar{u})(\nu-\bar{u}) d x \geq 0 \quad \forall v \in \mathbb{K}
\end{aligned}
$$

where I deftotes the identity matrix $N \times N$ and $\nabla \bar{y} \cdot \nabla \bar{y} I$ denores the $N \times N$ matrix with coefficients $\frac{\partial \vec{v}}{\partial x_{i}} \cdot \frac{\partial \bar{y}}{\partial x_{i}} l \leq i, j \leq N$.

Proof of this theorem requires a rather long development and it will be carry out in Sections 4 and 5 . 


\section{Remarks}

1) Remember that if $\alpha>2$ and $k=0$, it follows from the hypotheses that $b \in C^{1}(\Omega \times[0,+\infty))$ with $\frac{\partial b}{\partial s}(x, 0)=0$ and we apply the results of [3.5].

2) In the case $\alpha<2$ and $k=0$, we can obtain some additional information about the adjoint state $\bar{p}$;

$$
\nabla \bar{p}(x)=0 \quad \text { a.e } x \in \Omega \backslash \Omega_{0}
$$

See the end of section 5 for the prool.

3) Introduction of set $\Omega_{0}$ was suggested by the work [6] of $\mathrm{A}$. Friedman.

\section{AN APPROXIMATING FAMILY OF PROBLEMS $\left(P_{t r}^{c}\right)$}

Let $\bar{u}$ be a solution of $\left(P_{u r}\right)$ (see theorem 3.1). In order to derive the optimality system for $\bar{u}$. we introduce the following family of control problems:

$$
\left(P_{e}\right)\left\{\begin{array}{c}
\text { Minimize } J_{\mathrm{e}}(\nu) \\
\nu \in \mathcal{K}
\end{array}\right.
$$

where the cost functional is given by

$$
J_{2}(\nu)=\frac{1}{2} \int_{\Omega}\left|y_{\varepsilon}(v)-v_{v}\right|^{2} d x+\frac{\rho}{2} \int_{\Omega}|\nu|^{2} d x+\frac{1}{2} \int_{\Omega}|\nu-\bar{u}|^{2} d x
$$

and $r_{c}(\nu)$ is the solution of (2.2).

Following result can be proved arguing as in theorem 3.1, with the aid of lemma 2.5 .

Theorem 4.1. Let us suppose $(1.2)-(1.4),(1.6)-(1.7)$ and (1.9). Tho' for each $\varepsilon>0$, there exists (at least) one solution of $\left(I_{n}^{k}\right)$.

Before deriving the optimality conditions for $\left(I_{n}^{x}\right)$, we need to define some functional spaces.

Given $y \in W_{01}^{\prime a}(\Omega)$. let $W_{0}^{2, r}(\Omega)$ be the space completed of $D(\Omega)$ respect to the norm

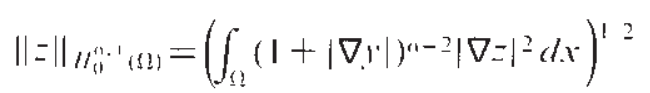


It may be easily verified that $H_{0}^{\prime \prime} \cdot{ }^{\prime \prime}(\Omega)$ is a Hilbert space with the inner product

$$
\left(z_{1}, z_{2}\right)=\int_{\Omega}(1+|\nabla y|)^{r-2} \nabla z_{1} \nabla z_{2} d x
$$

Moreover. we have

$$
\begin{aligned}
& W_{0}^{l, ~}{ }^{*}(\Omega) \subset H_{0}^{(r-b}(\Omega) \subset H_{0}^{l}(\Omega) \quad \text { if } \alpha \geq 2 \\
& H_{0}^{\prime}(\Omega) \subset H_{0}^{(r, b}(\Omega) \subset W_{0}^{\prime \cdot r}(\Omega) \quad \text { if } \alpha \leq 2
\end{aligned}
$$

with continuous imbeddings.

More general spaces of this type have been studied by Murthy and Stampacchia [9], Coffman et al. [4] and Trudinger [15].

Since operator $A_{c}$ satisfies the hypotheses of $[3,5]$ (see lemma 2.2), we deduce the following results which are analogue to [5. theorems 3.2 and 3.7 ].

Theorem 4.2. Let us suppose (1.2)-(1.4), (1.6)-(1.7) and one of the following conditions:

$$
\begin{array}{ll}
\text { i) } \alpha \geq 2, \alpha>N / 2 & \text { ii) } \alpha<2 \text { and } N=I
\end{array}
$$

For each $\varepsilon>0$, let $u_{\varepsilon}$ be a solution of $\left(P_{i r}^{\infty}\right)$ and $y_{\varepsilon} \in W_{0}^{l}$ " $(\Omega)$ the associated state. Then there exists a unique $p_{\varepsilon} \in H_{0}^{\left(*, l_{i}\right.}(\Omega)$ such that

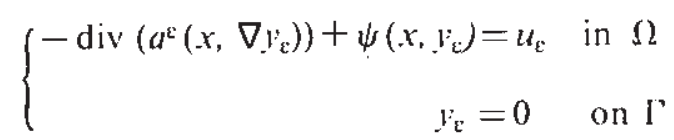

$$
\begin{aligned}
& \left\{\begin{array}{cc}
-\operatorname{div}\left(\frac{\partial a^{u}}{\partial \eta}\left(x, \nabla y_{c}\right) \nabla p_{c}\right)+\frac{\partial \psi}{\partial s}\left(x, y_{c}\right) p_{k}=y_{\varepsilon}-y_{d} & \text { in } \Omega \\
p_{c}=0 & \text { on } \mathrm{I}
\end{array}\right. \\
& \int_{\Omega}\left(p_{\mathrm{c}}+\rho u_{\mathrm{L}}+u_{\mathrm{L}}-\bar{u}\right)\left(\nu-u_{\varepsilon}\right) d x \geq 0 \quad \forall \nu \in \mathfrak{K}
\end{aligned}
$$

Theorem 4.3. Let us suppose (1.2)-(I.4). (1.6)-(I.7) and one of the following conditions:

$$
\text { i) } 1<\alpha<2 \text { and } N=2 \quad \text { ii) } \frac{3}{2}<\alpha<2 \text { and } N=3
$$


For each $c>0$, let $u_{\mathrm{\varepsilon}}$ be a solution of $\left(P_{t r}^{\mathrm{x}}\right)$ and $y_{\mathrm{\varepsilon}} \in W_{0}^{1}{ }^{\alpha}(\Omega)$ the associated state. Then there exists $p_{c} \in W_{10}^{\prime \prime}(\Omega)$ satisfying $(4.1)-(4.3)$ and

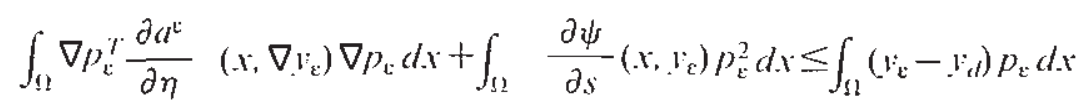

\section{Remarks}

1) There exists a unique solution in $H_{0}^{\alpha, r_{\mathrm{r}}}(\Omega)$ of problem $(4,2)$; it is enough to consider the bilinear form defined in $H_{0}^{\alpha . \mathrm{r}_{\mathrm{v}}}(\Omega)$ by

$$
B\left(z_{1}, z_{2}\right)=\int_{\Omega} \nabla z_{1}^{T} \frac{\partial a^{2}}{\partial \eta}\left(x, \nabla y_{1}\right) \nabla z_{2} d x+\int_{\Omega} \frac{\partial \psi}{\partial s}\left(x, y_{L}\right) z_{1} z_{2} d x
$$

and to apply the Lax-Milgram theorem.

2) In theorem 4.3 (case $\alpha<2$ and $N>1$ ), we can only prove that $p_{\text {: }}$ belongs to $W_{0}^{1, \alpha}(\Omega)$ and satisfies the equation in the distribution sense. In general we can not guarantee the uniqueness of $p_{c}$. In relation with this question see Serrin [11].

Before stating in what sense the problem $\left(P_{\alpha}\right)$ is approximated by the problems $\left(P_{\alpha}^{c}\right)$ we need to prove two previous lemmas:

Lemma 4.4. Let u.s suppose (1.2)-(1.4).(1.6)-(1.7) and (1.9). For each $\varepsilon>0$. let $\left(y_{c}\left(\nu_{\varepsilon}\right), \nu_{\varepsilon}\right)$ belong to $\left(W_{0}^{1.4 t}(\Omega) \cap L^{\infty}(\Omega)\right) \times L^{2}(\Omega)$ and satisfy

$$
\left\{\begin{aligned}
-\operatorname{div}\left(a^{\varepsilon}\left(x, \nabla y_{\varepsilon}\left(\nu_{v}\right)\right)+\psi\left(x, y_{\varepsilon}\left(\nu_{\varepsilon}\right)\right)=\nu_{\varepsilon}\right. & \text { in } \Omega \\
y_{c}\left(\nu_{\varepsilon}\right)=0 & \text { on } \Gamma
\end{aligned}\right.
$$

Let us assume that $\left\{w_{i}\right\}_{0}>0$ is bounded in $L^{2}(\Omega)$, then there exists $C>0$ such that

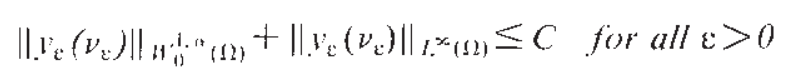

\section{Proof}

The boundedness of $\left\{I_{i}\left(\nu_{c}\right)\right\}_{c}>0$ in $W_{0}^{1}$, r $(\Omega)$ is a simple consequence of lemmas 2,3 and 2.4 . 
We will prove that $\left\{y_{k}\left(\nu_{x}\right)\right\}_{x>0}$ is bounded in $L^{\times}(\Omega)$.

First, given $r>0$, we consider

$$
r_{c}^{r}\left(\nu_{c}\right)=\max \left\{r_{\varepsilon}\left(\nu_{\mathrm{c}}\right)-r, 0\right\} \text { and } A_{\mathrm{c}}(r)=\left\{x \in \Omega: r_{\mathrm{c}}\left(\nu_{\mathrm{v}}\right)(x) \geq r\right\}
$$

We have $y_{n}^{\prime \prime}\left(\nu_{L}\right) \in W_{0}^{\prime \prime r}(\Omega)$ and

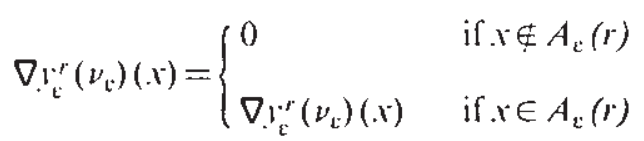

for almost every $x$ in $\Omega$.

Using Hölder's inequality and lemma 2.3 or lemma 2.4 , we deduce from (4.5) and (4.6) that

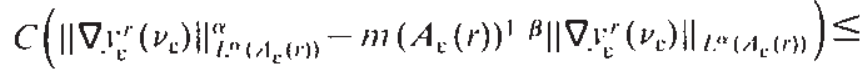

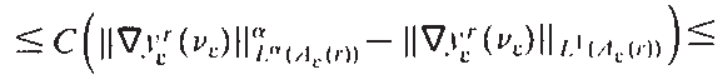

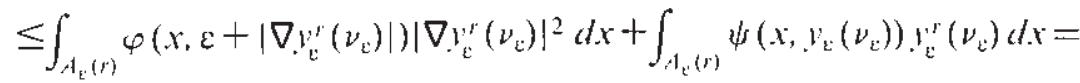

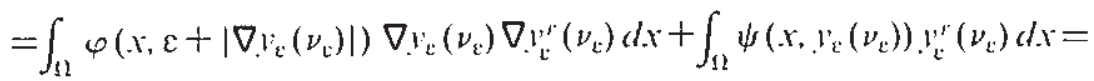

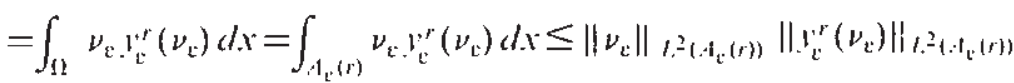

Thus, by the continuity of the imbedding $W_{0}^{1 . \alpha}(\Omega) \subset L^{\mu}(\Omega)$, with $\mu=\frac{N \alpha}{N-\alpha}$ (il $\alpha<N$ ) or $\mu>2 \alpha$ (if $\alpha=N$ ), see Adams [1], and the hypothesis. we get

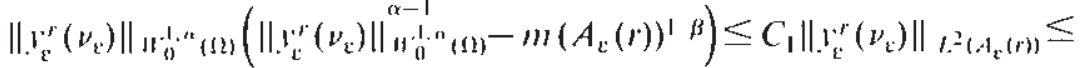

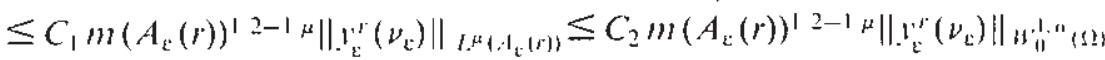

and hence,

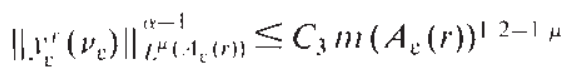

Now. let $s>r>0$; then $A_{\mathrm{L}}(s) \subset A_{\mathrm{r}}(r)$ and moreover

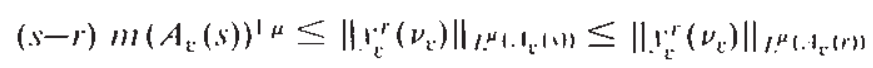


From (4.7) and (4.8) it follows

$$
m\left(A_{\varepsilon}(s)\right) \leq \frac{C}{(s-r)^{\mu}} m\left(A_{\varepsilon}(r)\right) \frac{\mu}{\alpha-1}\left(\frac{1}{2}-\frac{1}{\mu}\right)
$$

Finally, applying lemma 4.I of Stampacchiat [12] to the function $\phi_{\mathrm{r}}(t)=m\left(A_{\varepsilon}(t)\right)$ for $1>0$. and noting that thanks to (1.9)

$$
\frac{\mu}{\alpha-1}\left(\frac{1}{2}-\frac{1}{\mu}\right)>1
$$

it follows the existence of a constant $C_{5}<+\infty$ independent of 2 such that $r_{i}\left(u_{k}\right)(x) \leq C_{5}$ a e.e $x \in \Omega$.

In the same way, taking. $y^{\prime \prime}\left(v_{\varepsilon}\right)=\min \left\{r_{2}\left(v_{\varepsilon}\right)+r, 0\right\}$, we derive the existence ol $C_{n}>-\infty$ such that $l_{\varepsilon}\left(\nu_{n}\right)(x) \geq C_{n}$ a.e. $x \in \Omega$ tor every $\varepsilon>0$.

lemma 4.5. Let as stuplose (1.2)-(1.4). (1.6)-(1.7) and (1.9). Assumb

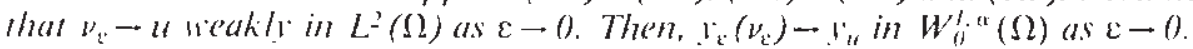

\section{Proof}

From previous lemma we derive that $\left\{f_{t}\left(\nu_{c}\right) h_{n>0}\right.$ is bounded in $W_{0}^{1}$ "r $(\Omega)$. Thus. there exist a subsequence (again denoted $\left.\left\{v_{2}\left(\nu_{2}\right)\right\}_{2>0}\right)$ and $y \in W_{0, "}^{\prime \prime}(\Omega)$ such that

$$
l^{*}\left(\nu_{n}\right)-y^{*} \quad \text { weakly in } W_{0}^{\prime a c}(\Omega)
$$

Furthermore, $I_{c}\left(\nu_{v}\right)$ is the solution of $(2.2)$ with $v=\nu_{\varepsilon}$ and then we have

$\int_{12} \varphi\left(x, \varepsilon+\left|\nabla_{1}\left(\nu_{k}\right)\right|\right) \nabla_{1}\left(\nu_{k}\right) \nabla \phi d x+\int_{n} \psi\left(x, l_{2}\left(\nu_{k}\right)\right) \phi d x=\int_{1 !} \nu_{k} \phi d x$

for all $\phi \in H_{0}^{\prime} \cdot(1)$

For proving that $y=y_{\text {" }}$ it is sufficient to pass to the limit in $(4.9)$ ats $\varepsilon \rightarrow 0$.

In virtue of b). d) of lemma 2.3 and lemma 4.4 we have

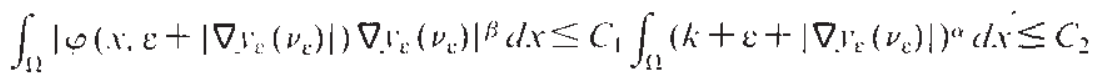

$$
\begin{aligned}
& \int_{! !}\left|\psi\left(x, r_{n}\left(\nu_{v}\right)\right)\right|^{\beta} d x \leq C_{3}
\end{aligned}
$$


Therefore, we may infer that there exist a subsequence (denoted in the same way) and $\chi \in\left(L^{\beta}(\Omega)\right)^{N}$ such that

$$
\begin{aligned}
& \varphi\left(x, \varepsilon+\left|\nabla y_{\varepsilon}\left(\nu_{\varepsilon}\right)\right|\right) \nabla v_{\varepsilon}\left(\nu_{\varepsilon}\right) \rightarrow \chi \quad \text { weakly in }\left(L^{\beta}(\Omega)\right)^{*} \\
& \psi\left(x, y_{c}\left(\nu_{c}\right)\right) \rightarrow \psi(x, y) \quad \text { weakly in } L^{\beta}(\Omega)
\end{aligned}
$$

Let us introduce the element $L$ of $W-1, \beta(\Omega)$ defined by the formula

$$
L(\phi)=\int_{\Omega} x \nabla \phi d x
$$

Letting $\varepsilon$ tend to 0 in $(4,9)$ we deduce

$$
L(\phi)=\int_{11}(u-\psi(x, v)) \phi d x
$$

for all $\phi \in W_{0}^{1, \alpha}(\Omega)$. Moreover, by (4.10) and the strong convergence of $y_{\mathrm{c}}\left(\nu_{\varepsilon}\right)$ to $y$ in $L^{2}(\Omega)$ and $L^{\alpha}(\Omega)$

$$
\begin{gathered}
\limsup _{\varepsilon}\left(\int_{\Omega} \varphi\left(x, c+\left|\nabla y_{\varepsilon}\left(\nu_{\varepsilon}\right)\right|\right)\left|\nabla y_{c}\left(v_{\varepsilon}\right)\right|^{2} d x\right)= \\
=\limsup _{\varepsilon}\left(\int_{\Omega} \nu_{\mathrm{c} .} \nu_{\mathrm{\varepsilon}}\left(\nu_{\varepsilon}\right) d x-\int_{\Omega} \psi\left(x, v_{c}\left(v_{\varepsilon}\right)\right) y_{\mathrm{c}}\left(\nu_{\mathrm{c}}\right) d x\right)= \\
=\int_{\Omega}(u-\psi(x, y)) y d x=L(v)
\end{gathered}
$$

Since the operator $Y: W_{0}^{1, \alpha}(\Omega) \rightarrow W^{-1, \beta}(\Omega)$ defined by

$$
(\curvearrowright y, w)=\int_{n} \varphi(x,|\nabla y|) \nabla y, \nabla w d x
$$

satisfies $M$-property (Lions [8, pp. 171-187]) and $\gamma_{b_{\mathrm{c}}}\left(\nu_{\mathrm{v}}\right)-L$ weakly in $W^{-i, \beta}(\Omega)$, it is verified that

$$
\int_{\Omega} \varphi\left(x,\left|\nabla_{y}\right|\right) \nabla_{!} \nabla \phi d x+\int_{\Omega} \psi(x, y) \phi d x=\int_{\Omega} u \phi d x \quad \forall \phi \in W_{0}^{l} \cdot a(\Omega)
$$

Hence, $y=y_{t}$ and then

$$
y_{c}\left(\nu_{c}\right) \rightarrow y_{t} \quad \text { weakly in } W_{0}^{1, x}(\Omega)
$$

Finally, from the above results and lemma 2.3, we conclude in the case $\alpha \geq 2$ that

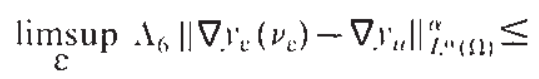




$$
\begin{aligned}
& \leq \limsup _{\varepsilon} \int_{\Omega}\left(\varphi\left(x, \varepsilon+\left|\nabla_{l_{c}}\left(\nu_{c}\right)\right|\right) \nabla_{v_{c}}\left(\nu_{c}\right)-\varphi\left(x, c+\left|\nabla_{y_{u}}\right|\right) \nabla_{y_{u}}\right)\left(\nabla_{l_{\llcorner}}\left(\nu_{c}\right)-\nabla_{y_{t}}\right) d x=
\end{aligned}
$$

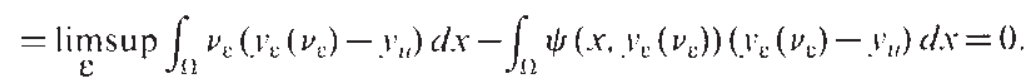

In the case $\alpha<2$, the argument is the same, utilizing lemma 2.4 .

Hence, $y_{i}\left(v_{\varepsilon}\right) \rightarrow y_{\text {ti }}$ strongly in $W_{i}^{\text {l. a }}(\Omega)$, as $\varepsilon \rightarrow 0$.

Theorem 4.6. Let us suppose (1.2)-(1.4). (1.6)-(1.7) and (1.9). Let ute be a solution of $\left(P_{v}^{\mathrm{c}}\right)$. Set $\bar{l}=\mathrm{y}_{\bar{u}}$ and $v_{\mathrm{c}}=\mathrm{y}_{\mathrm{c}}\left(u_{\mathrm{v}}\right)$. Then, we have

$$
\begin{gathered}
u_{\varepsilon} \rightarrow \bar{u} \text { in } l^{2}(\Omega) \\
l_{\varepsilon} \rightarrow \bar{y} \text { in } W_{0}^{1 \cdot u}(\Omega) \\
J_{\varepsilon}\left(u_{\varepsilon}\right) \rightarrow J(\bar{u})
\end{gathered}
$$

as $\varepsilon \rightarrow 0$.

\section{Proof}

Applying previous lemma to $\nu_{\varepsilon}=\bar{u} \quad \forall \varepsilon>0$. we deduce

$$
r_{v}(\bar{u}) \rightarrow \bar{y} \text { in } W_{i}^{\prime} \cdot r(\Omega)
$$

Since $\pi \in \mathcal{K}$. it follows that for all $\varepsilon>0$

$$
{ }_{2}\left\|u_{v}-\bar{u}\right\|_{i, 2(s)}^{2} \leq J_{v}\left(u_{\varepsilon}\right) \leq J_{i}(\bar{u}) \leq C
$$

Thus, $\left\{u_{v}\right\}_{2}>0$ is bounded in $L^{2}(\Omega)$ and selecting a subsequence, if necessary, we may infer that there exists $u \in \mathcal{K}$ such that

$$
u_{\varepsilon} \rightarrow U \text { weakly in } L^{2}(\Omega)
$$

Using once more lemma 4.5, we obtain

$$
l_{i} \rightarrow y_{t \prime} \text { in } W_{0}^{1, t}(\Omega)
$$

From (4.14) and the lower semicontinuity of $J_{v}$, we get

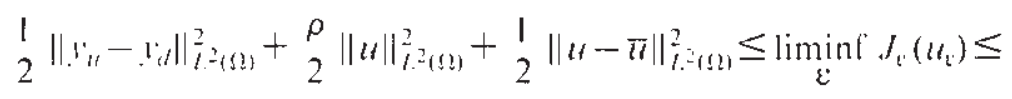




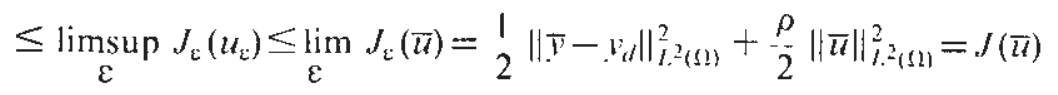

Since $\bar{u}$ is solution of $\left(P_{v}\right)$, we know that $J(\bar{u}) \leq J(u)$, thus $u=\bar{u}$ and

$$
J_{i}\left(u_{i}\right) \rightarrow J(\bar{u}) \quad \text { as } \varepsilon \rightarrow 0
$$

Moreover, $u_{\mathrm{c}}$ converges strongly towards $\bar{u}$, because

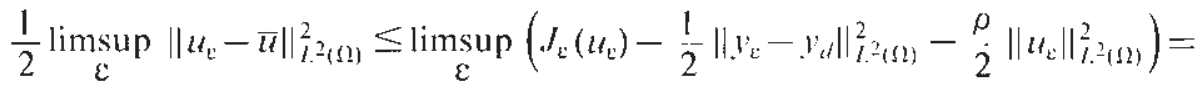

$$
\begin{aligned}
& =\frac{\rho}{2}\left(\|\bar{u}\|_{i^{2}(1,2)}^{2}-\liminf \left\|u_{i}\right\|_{i^{2}(0)}^{2}\right)=0 .
\end{aligned}
$$

\section{PROOF OF THEOREM 3.2}

In the preceding section, given $\bar{u}$ a solution of $\left(P_{r}\right)$, we have obtained the optimality conditions for the solutions of the perturbed problems. Hereafter, our purpose is to pass to the limit in conditions $(4.1)-(4.3)$ with the aid of the last results. We will distinguish two cases:

\subsection{Case $\alpha \geq 2$ and $k \neq 0$}

Let $\left\{D_{\mathrm{e}}\right\}_{\mathrm{r}}>010 H_{0}^{\alpha, I_{\mathrm{x}}}(\Omega) \subset H_{0}^{\prime}(\Omega)$ be given as in theorem 4.2 .

Applying (4.2) to $p_{\varepsilon}$, we derive

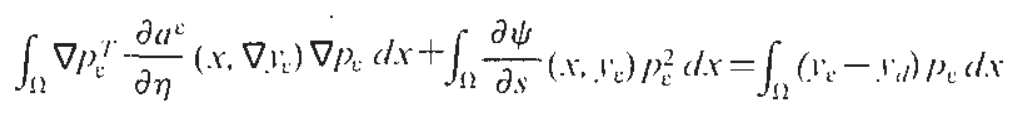

By lemma 2.2. (1.6) and Hölder's inequality, we have

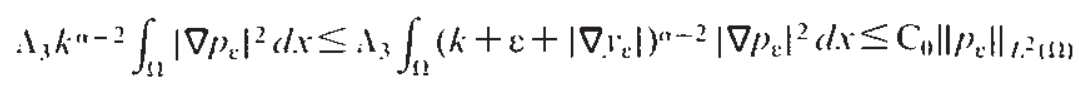

In particular, since $k \neq 0$, it follows from (5.1) that $\left\{p_{k}\right\}_{k=10}$ is a bounded sequence in $H_{,}^{\prime}(\Omega)$ and there exist a subsequence (again denoted $p_{2}$ ) and an element $\bar{p} \in H_{0}^{\prime}(\Omega)$ such that

$$
p_{\varepsilon} \rightarrow \bar{p} \text { weakly in } H_{i}^{\prime}(\Omega)
$$

It is immediate to obtain (3.3) taking into accoun (4.11) and patssing 10 the limit in $(4.3)$ as $\varepsilon-0$. 
It remains to verify that $\bar{p}$ satisfies equation (3.2):

Since $\pi \in \mathcal{H} \subset L^{\infty}(\Omega)$ and thanks to lemma 2.2, we can apply a Tolksdorf's result $[13]$ and deduce that the optimal state $\bar{F}$ belongs to $C^{l} . \mu(\Omega)$ for some $0<\mu<1$. Therefore, $\Omega_{0}=\{x \in \Omega:|\nabla \bar{v}(x)|>0\}$ is an open set of $\mathcal{H}^{N}$.

Let $\phi \in D\left(\Omega_{0}\right)$ and let us denote sop $\phi=\bar{\Omega}^{\prime} \subset \Omega$. Our purpose is to pass to the limit as $\varepsilon \rightarrow 0$ in the following expression

$$
\int_{\Omega L} \nabla \phi^{r} \frac{\partial \underline{a}^{\mathrm{L}}}{\partial \eta}\left(x, \nabla_{y_{\varepsilon}}\right) \nabla p_{\varepsilon} d x+\int_{\Omega} \frac{\partial \psi}{\partial s}\left(x, y_{\varepsilon}\right) p_{k} \phi d x=\int_{\Omega}\left(y_{\varepsilon}-v_{i}\right) \phi d x
$$

Utilizing again the Tolksdorf's result [13], it follows the existence of constants $C_{1}$ and $C_{2}$ depending only on $N, \alpha, \Lambda_{1}, \Lambda_{2}, d\left(\Omega^{\prime}, \Gamma\right),\left\|u_{\varepsilon}\right\|_{1^{\infty}(\Omega)}$ and $\left\|v_{\varepsilon}\right\|_{l^{x}(1 ! 1)}$ such that

$$
\begin{aligned}
\left|\nabla_{y_{\mathrm{r}}}(x)\right| & \leq C_{1} \quad \forall x \in \Omega^{\prime} \\
\left|\nabla_{y_{\varepsilon}}(x)-\nabla y_{\mathrm{\varepsilon}}\left(x^{\prime}\right)\right| & \leq C_{2}\left|x-x^{\prime}\right|^{\mu} \quad \forall x, x^{\prime} \in \Omega^{\prime}
\end{aligned}
$$

By hypotheses, $\mathbb{J}$ is bounded in $L^{\infty}(\Omega)$. Furthermore, we know that $\left\|b_{\varepsilon}\right\|_{l^{\times}(\Omega)}$ is uniformly bounded by a constant independent of $\varepsilon$ (see lemma 4.4). Then, we can apply Ascoli-Arzelá theorem to deduce the existence of a subsequence (denoted in the same way) such that

$$
\nabla y_{c}(x)-\nabla \bar{y}(x) \text { uniformly in } \bar{\Omega}
$$

By other hand, in virtue of temma 2.2 and taking $\varepsilon \in(0.1)$ we obtain that $\left\|\frac{\partial q^{*}}{\partial \eta}\left(x, \nabla y_{c}\right)\right\| \leq \Lambda_{5}\left(k+\varepsilon+\left|\nabla y_{c}\right|\right)^{k-2} \leq \Lambda_{5}\left(k+1+C_{1}\right)^{r-2} \quad \forall x \in \Omega^{\prime}$

Taking into account $(1,6)$ and lemma 4.4 , it follows that

$$
\left|\frac{\partial \psi}{\partial y}\left(x, r_{c}\right)\right| \leq f\left(\left\|y_{\varepsilon}\right\|_{l^{*}(I,)}\right) \leq C_{3} \quad \forall x \in \Omega \quad \forall \varepsilon>0
$$

Thanks to the hypothesis (1.3), lemma 2.2 , convergence (5.4), (4.12) and the Dominated Convergence Theorem, we deduce that as $\varepsilon-0$

$$
\begin{array}{cl}
\frac{\partial \psi}{\partial s}\left(x, y_{i}\right)-\frac{\partial \psi}{\partial s}(x, \bar{y}) & \text { in } L^{\prime}(\Omega) \\
\frac{\partial u^{2}}{\partial \eta}\left(x, \nabla_{b_{c}}\right) \rightarrow \frac{\left.\partial u^{i}\right)}{\partial \eta}\left(x, \nabla_{\bar{y}}\right) & \text { in }\left(L^{\prime}\left(\Omega^{\prime}\right)\right)^{N \times N}
\end{array}
$$

for all $1 \leq r<+\infty$.

Now. we can pass to the limit in (5.3) with the aid of (5.2) and $(4.12)$. 


\subsection{Case $\alpha<2$}

Let $\left\{p_{1:}\right\} \subset W_{1}^{l}{ }^{\alpha}(\Omega)$ be given as in theorem 4.2 (if $N=1$ ) or theorem 4.3 (if $N>1)$

Using the same argument as in the proof of lemma 2.4-a) and the conclusion of lemma 4.4, we get

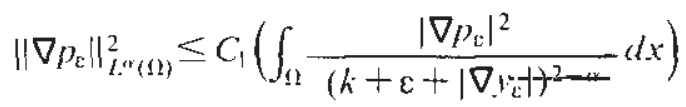

Combining lemma $2.2-$ a), (1.6), (5.5) and taking into account that $p_{x}$ is solution of (4.2) if $N=1$ or using (4.4) if $N>1$, we deduce

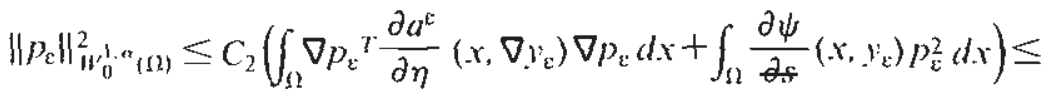

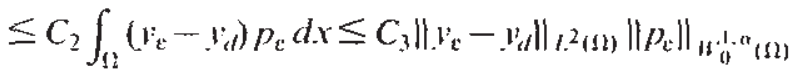

Remind that $W_{0}^{1 .}{ }^{\circ}(\Omega) \subset L^{2}(\Omega)$ thanks to (1.9).

Thus, $\left\{p_{\varepsilon}\right\}_{\varepsilon}>0$ is bounded in $W_{0}^{1 . \alpha}(\Omega)$ and it is possible to choose a subsequence $\varepsilon(n) \rightarrow 0$ such that

$$
p_{\varepsilon(n)} \rightarrow \bar{p} \quad \text { weakly in } W_{i j}^{\prime . r}(\Omega)
$$

for some $\bar{p} \in W_{0}^{1} \alpha(\Omega)$.

Rest of the theorem follows exactly as in the previous proof.

We conclude this section proving the following additional property about $\bar{p}$ in the case $\alpha<2$ and $k=0$ :

$$
\nabla \bar{p}(x)=0 \quad \text { a.e. } x \in \Omega \backslash \Omega_{0}
$$

Let $K$ be a compact subset of $\Omega \backslash \Omega_{0}$. Then, we know that

$$
\nabla v_{\mathrm{c}}(x) \rightarrow \nabla \overline{I^{\prime}}(x)=0 \quad \text { uniformly in } K
$$

Given $\delta>0$, there exists $\varepsilon^{\prime}>0$ such that

$$
\left(\varepsilon+\left|\nabla I_{L}(x)\right|\right)^{2-\infty}<\delta \quad \forall \varepsilon<\varepsilon^{\prime} \quad \forall x \in K
$$


Now, thanks to (5.5), we deduce

$$
\frac{1}{\delta} \int_{K}\left|\nabla p_{\varepsilon}\right|^{2} d x \leq \int_{K} \frac{\left|\nabla p_{i}\right|^{2}}{\left(\varepsilon+\left|\nabla \bar{r}_{i}\right|\right)^{2}-x} d x \leq C_{4}
$$

and we can conclude that

$$
\nabla P_{\mathrm{c}(n)} \rightarrow \nabla \vec{p} \quad \text { weakly in }\left(L_{*}^{2}(K)\right)^{k}
$$

Hence, we have

$$
\int_{K}|\nabla \bar{p}|^{2} d r \leq \liminf _{\|} \int_{K^{\prime}}\left|\nabla p_{x, m}\right|^{2} d r \leq C_{4} \delta \quad \forall \delta>0
$$

which implies $\nabla \bar{p}(x)=0$ a.e. $r \in K$.

Since $\Omega \backslash \Omega_{0}$ can be written as a countable union of compact sets (except a set of measure zero). assertion (5.7) holds.

\section{REGULARITY OF THE OPTIMAI, CONTROI,}

In this last section, we deduce some qualitative properties about optimal controls. using the optimality conditions.

Theorem 6.1. Les us suppose that $\rho$ is strictly positive in (l.11) and

$$
\begin{gathered}
\mathcal{K}=\left\{\nu \in L^{2}(\Omega): m \leq \nu(x) \leq M \text { a.e.x } \in \Omega\right\} \\
\text { with }-\infty<m<M<+\infty \text {. Then, } \bar{u} \in \begin{cases}H^{\prime}(\Omega) & \text { if } \alpha \geq 2 \\
W^{\prime . t}(\Omega) & \text { if } \alpha<2\end{cases}
\end{gathered}
$$

\section{Proof}

Inequality $(3,3)$ characterizes $\bar{H}$ as the projection of $-\frac{\bar{P}}{\rho}$ on $\mathcal{K}$. Hence, it follows that

$$
\bar{t}(x)=\max \left\{m . \min \left\{-\frac{1}{\rho} \bar{p}(x) . M\right\}\right\} \quad \text { a.e. } x \in \Omega
$$

We conclude the proof noting that function $f: \mathfrak{R} \rightarrow \mathfrak{R}$ defined by

$$
f(s)=\max \{m, \min \{s, M\}\}
$$


is uniformly Lipschitz, using lemma 28.1 of Treves [14, pg. 261] and the fact that $\bar{p} \in\left\{\begin{array}{ll}H_{0}^{1}(\Omega) & \text { if } \alpha \geq 2 \\ W_{0}^{1, \alpha}(\Omega) & \text { if } \alpha \leq 2\end{array}\right.$.

\section{Remarks}

1) If $0 \in[m, M]$, moreover we obtain that $\bar{u}_{I^{\prime}}=0$.

2) If $\rho=0$, it follows from (3.3) that

$$
\begin{cases}\bar{u}(x) \in[m, M] & \text { if } \bar{p}(x)=0 \\ \bar{u}(x)=m & \text { if } \bar{p}(x)>0 \\ \bar{u}(x)=M & \text { if } \bar{p}(x)<0\end{cases}
$$

When $\bar{p}(x) \neq 0$ a.e. $x \in \Omega$, we have that $\bar{u}$ is ubang-bang".

In the conditions of theorem 6.1 and if $N=1$, the Hölder continuity of $\bar{u}$ in $\bar{\Omega}$ is a consequence of the Sobolev imbedding theorem. In general $(N>1)$, assuming that $y_{t} \in L^{p^{\prime}}\left(\Omega_{0}\right)$ with $\rho^{\prime}>N / 2$, we can apply Theorem 14.1 of Ladyzhenskaya-Ural'tseva $[7$, p. 201$]$ and deduce that $\bar{p}$ is Hölder continuous in $\Omega_{0}$ (and then $\bar{u}$ too), see $[3,5]$.

\section{References}

1. R. A. ADAms: Sobolew Spaces. Academic Press. New York. 1975.

2. E. CASAS and L. A. FERNAN[B:z: Optimal control of quasilifear elliplic equarions, in A. Bermúdez, editor, "Control of Partial Differential Equations". L.ecture Notes in Control \& Inform. Sci. 114 (1989), 92-99, Springer-Verlalg.

3. E. CASAS and L. A. FFRNANDEZ: Distributed control of swstems governed by a general class of quasilinear elliphic equations. J. Differential Equations. To appear.

4. C. V. Coffman, V. DufFin and V. J. M1ZFi.: Positivity of weak solutions of non-thiform!y elliplic equations. Ann. Mat. Pura Appl. 104 (1975). 209-238.

5. [.. A. FERNANDLZ: Control ómimo de sistemas gobemados por ecuaciones elipricas ctasilineales, Doctora] Thesis, Universidad de Cantabria, 1990.

6. A. FRILDMAN: Optimal comtrol for variational istequalities. SIAM J. Control Optim. 24 (1986). 439-45I.

7. O. A. LADYZHENSKAYA and N. N. URAL"TSEVA: Linear and Quasilinear elliptic cquations, Academic Press, New York and London, 1968.

8. I. I.. I.LONs: Quelques Méthodes de résolution des problèmes aux limites non lincaires. Dunod. Paris. 1969. 
9. M. K. V. MURTHY and G. STAMPACCHA: Bundary value problems for some degenerate elliptic operators, Ann. Mat. Pura Appl. 80 (1968), 1-122.

10. J. M. RAKoroson: Réarrangement relatif dans les ćquations elliptiques quasilinéaries avec un second membre distribution: Application a tan théorème d'existence et de régularité. J. Differential Equations 66 (1987), 391-419.

11. J. SI:RRIN: Pathological solutions of ellipic differemial equations. Anss. Scuola Norm. Sup. Pisa 18 (1964), 385-387.

12. G. SэлмгАсСнал: Le problème de Dirichlet pour les équations ellipriques dh second ordre à coefficients discontinus, Ann. Inst. Fourier 15 (1965), 189-258.

13. P. TOLKSDORF: Regularisy for a mowe general class of quasilinear elliptic equations, I. Differential Equations 5I (1984). 126-150.

14. F. TRlives: Basic linear parlial differemtial equations, Academic Press. New York, 1975

15. N. S. Trudinger: Linear elliptic equations with measurable coefficients, Ann. Scuola Norm. Sup. Pisa 27 (1973), 265-308.

Departamento de Matemáticas. Fatadislica y. Computación

Universidad de Canlabria

39071 Siantilnder

Recibido: 6 de septiembre de 1490

SPAIN

Revisado: 7 de cnero de 199 